\title{
The rationale of mandible reconstruction in advanced oral cancer: alloplastic material versus autogenous vascularized bone graft
}

\author{
Tim-Mo Chen a , Hsian-Jenn Wang ', Tian-Yeu Cheng a, Ke-Chung Chang ${ }^{b}$, Feng-Huei Lin ${ }^{c, *}$ \\ ${ }^{a}$ Division of Plastic Surgery, Department of Surgery, Tri-Service General Hospital, National Defense Medical Center, Taipei, Taiwan \\ ${ }^{\mathrm{b}}$ Taiwan Adventist Hospital, Taipei, Taiwan \\ ${ }^{c}$ Institute of Biomedical Engineering, National Taiwan University, Taipei, Taiwan
}

\begin{abstract}
Autogenous vascularized bone grafts in combination with microsurgical techniques have revolutionized mandibular reconstruction in oral cancer surgery. The vascularized bone graft carries its own blood supply, resists infection, and heals rapidly even in the irradiated bed. However, its use in advanced oral cancer patients is controversial due to the gloomy prognosis of these patients. Effective alloplastic materials such as titanium plate or tray for mandibular reconstruction is a reasonable alternative. These can easily be shaped and have sufficient structural rigidity to maintain the mandibular fragments in proper orientation. Likewise, it is a fast and reliable procedure which can obtain good oral function. However, its potential late exposure due to breakdown of overlying skin, especially after radiotherapy, is well documented. This paper discusses our experience to rescue the oral function after alloplastic material failure using the vascularized fibula osteocutaneous flap, in irradiated patients. All fibula osteocutaneous flaps survived. Symmetry and contour of the reconstructed mandible were judged to be good, and patients were able to eat without saliva drooling. Donor-site morbidity was also low. From this clinical study, we believe that in advanced oral cancer, the titanium plate or tray is a useful adjunct for immediate mandibular reconstruction after surgical resection. The vascularized bone graft should be reserved for patients with alloplastic material failure. This rationale can avoid subjecting the patient to an unnecessary extensive mandibular reconstructive procedure. (C) 2000 Elsevier Science S.A. All rights reserved.
\end{abstract}

Keywords: Autogenous vascularized bone graft; Alloplastic materials

\section{Introduction}

The U-shaped mandible serves as the arch of the oral cavity, supporting the tongue and muscles of the mouth floor, thus permitting mastication, articulation, deglutition, and respiration. In addition, the mandible comprises much of the soft tissue bulk of the lower face. Therefore, primary reconstruction of the mandible defect after surgical ablation for cancer is imperative to maintain function, provide an acceptable facial contour, and enable the patient to achieve a reasonable quality of life with early oral functional rehabilitation.

In those cases where preoperative or postoperative radiotherapy are to be included in the treatment, continually

\footnotetext{
* Corresponding author. Tel.: +886-2-23970800; fax: +886-223940049.

E-mail address: double@ha.mc.ntu.edu.tw (F.-H Lin).
}

poor results of graft failure resulting from a poorly vascularized bed has led surgeons to abandon the free nonvascularized bone graft in primary mandibular reconstruction $[1,2]$. The host bed which requires either preoperative or postoperative irradiation is hypovascular, hypocellular, and hypoxic. The entire non-vascularized bone graft is often resorbed with soft tissue infection or breaks down due to the poor vascularity of the bed. Many of these problems were overcome with the advent of microvascular transfer of vascularized bone grafts [3-9]. The vascularized bone graft carries its own blood supply, resists infection, and heals readily even in the irradiated bed. Many donor-sites for vascularized bone graft are currently available, giving the surgeon a variety of choices. Many surgeons have embraced vascularized bone graft as the answer to primary mandibular reconstruction after cancer ablation surgery.

However, to use free vascularized bone graft in all mandibulectomy patients after cancer ablation surgery is 
not always necessary. Even with advances in surgical ablation, radiotherapy, and chemotherapy, oral cancer patients have an overall 5-year survival of only $40 \%$. This raises the question of whether or not patients with advanced malignancy or in poor physical condition should undergo a complicated microvascular procedure with a very limited life expectancy.

One of the alternatives is the use of the titanium tray or plate for mandibular reconstruction, provided that adequate soft tissue is used to cover the metal and line the oral cavity [10-16]. The titanium tray and plate are easily shaped and have sufficient structural rigidity to maintain the mandibular fragments in proper orientation. Generally, this method is indicated in cases of advanced disease or poor physical conditions. It is a fast and reliable procedure which can maintain good oral function and cosmetic results. However, exposure of hardware in an irradiated bed still remains a potential problem. Once exposed, the hardware usually has to be removed, which will result in invalid oral function. This paper presents our experiences

(a)

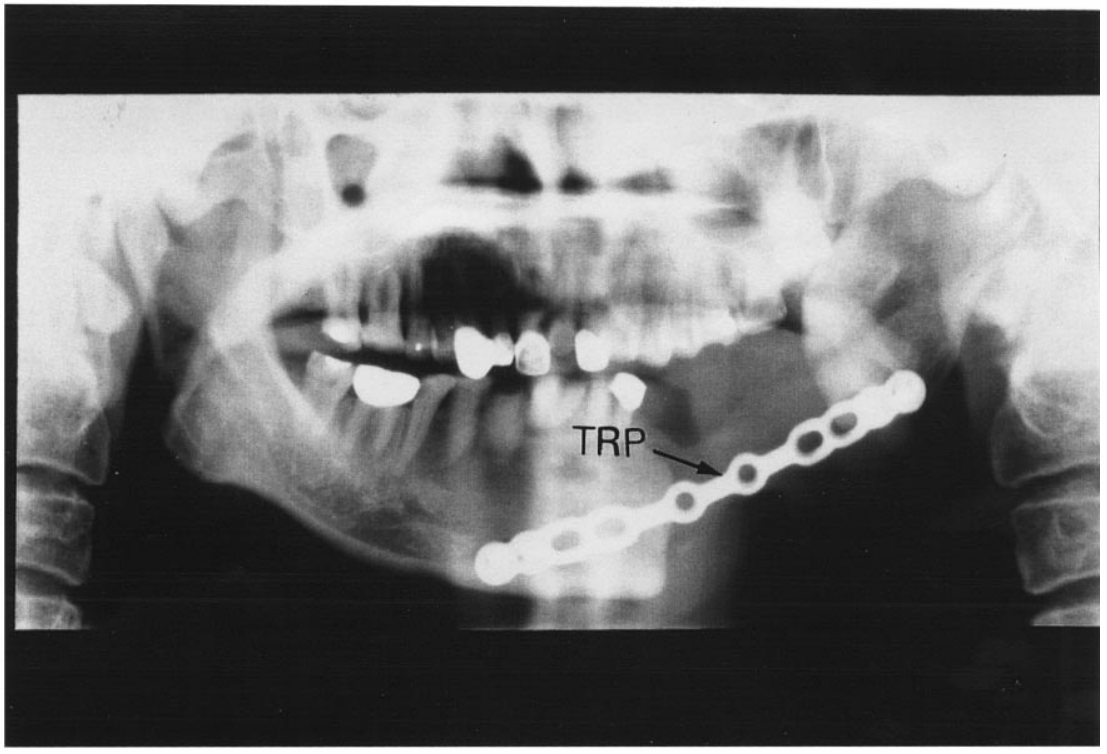

(b)

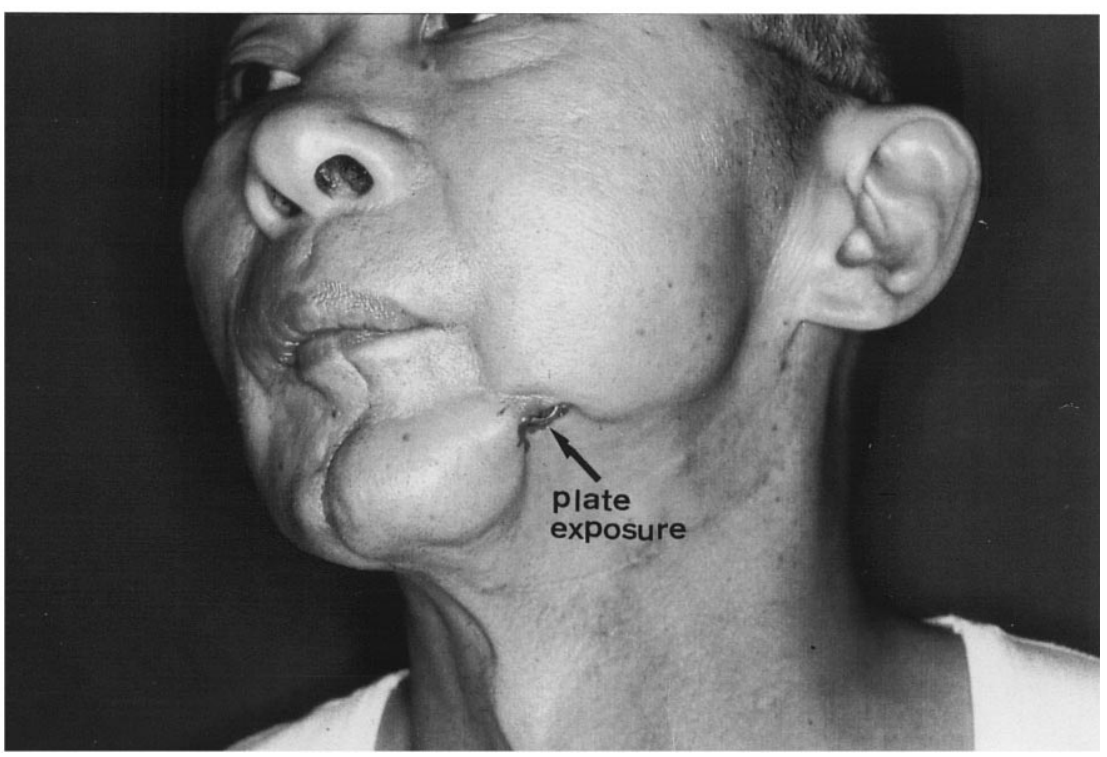

Fig. 1. (a) A patient with poorly differentiated epidermoid carcinoma of the mouth floor. Initial reconstruction of the mandible with a titanium reconstruction plate. TRP: titanium reconstruction plate. (b) Exposure of hardware (Arrow), 18 months after initial surgery. (c) Autogenous fibula osteocutaneous flap was harvested. AOF: Autogenous fibula osteocutaneous flap. (d) The fibula was osteomized to fit the remaining mandible and fixed with miniplates and screws. The skin island was used to resurface the external skin defect. MPS: Miniplates and screws. (e) Postoperative uneventful healing of the skin island. (f) Postoperative X-ray showed primary healing of the bone graft. 
(c)

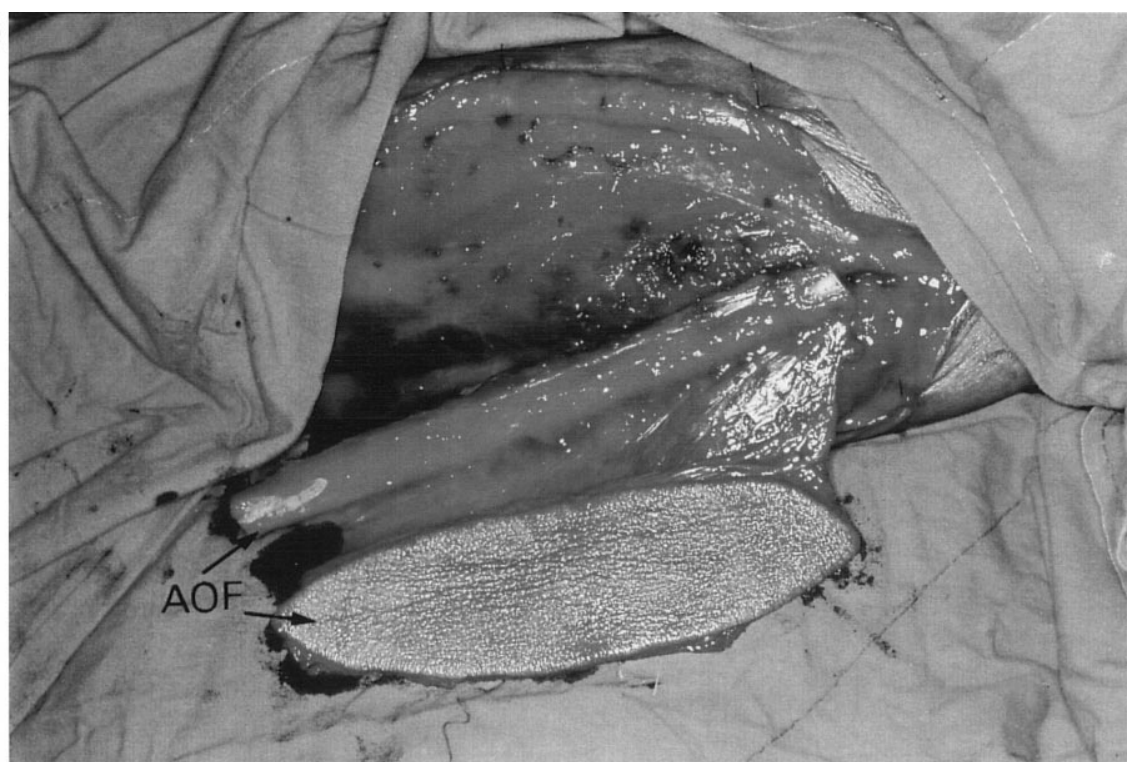

(d)

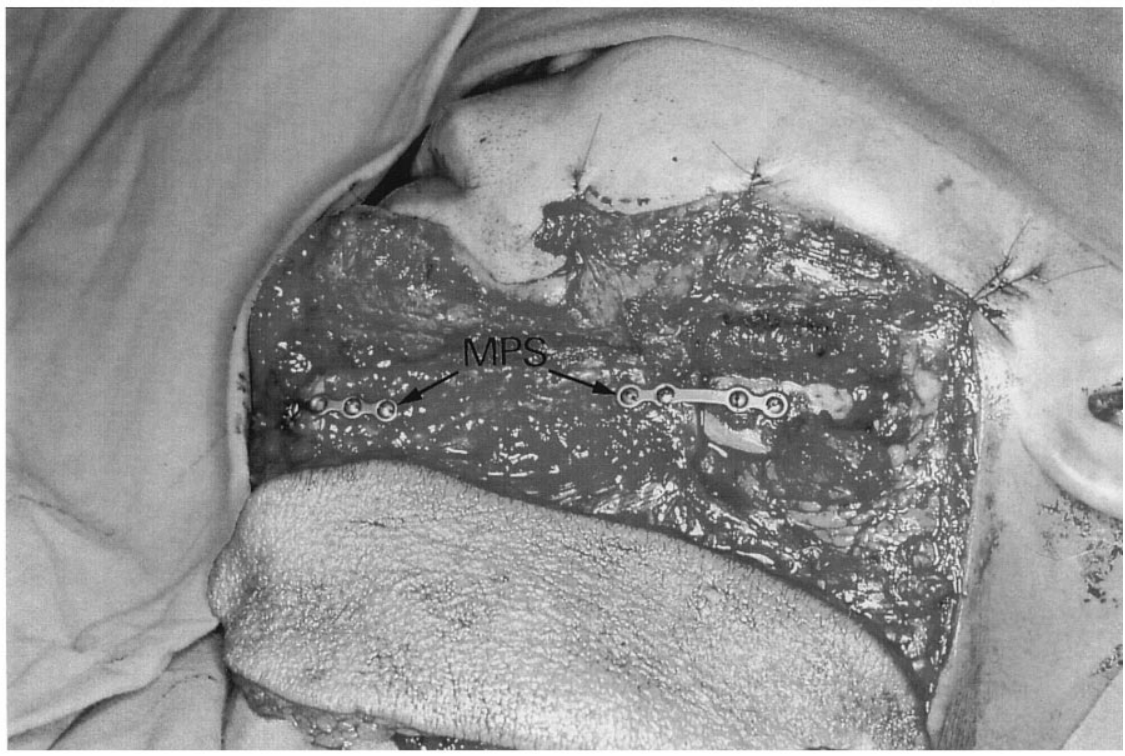

Fig. 1 (continued).

to rescue the oral function after hardware removal using a vascularized fibula osteocutaneous flap and presents an overview of the optimal use of this technique in mandibular reconstruction after cancer ablation surgery.

\section{Materials and methods}

\subsection{Dissection of the fibula osteocutaneous flap}

The dissection is performed with the patient in the supine position and the donor knee flexed. Dissection is by means of lateral approach under tourniquet according to the technique described by Wei et al. [17]. The designed skin flap centers at the junction between the middle and lower third of the lower leg. A minimal skin flap $4 \mathrm{~cm}$ in width and $8-10 \mathrm{~cm}$ in length is recommended, because a flap too small may miss the septocutaneous perforators which are the blood supply to the skin flap. Most of the fibula is harvested regardless of the length of the mandible requiring reconstruction. This facilitates the dissection of the vascular pedicle and maximizes the available length of the vascular pedicle. The distal $5 \mathrm{~cm}$ of the fibula should be preserved to maintain ankle stability. The peroneal artery and vein are divided and ligated distally.

\subsection{Shaping the fibula bone graft}

Measurements taken from the surgical specimen or templates fashioned from X-ray studies assist in accurately 
(e)

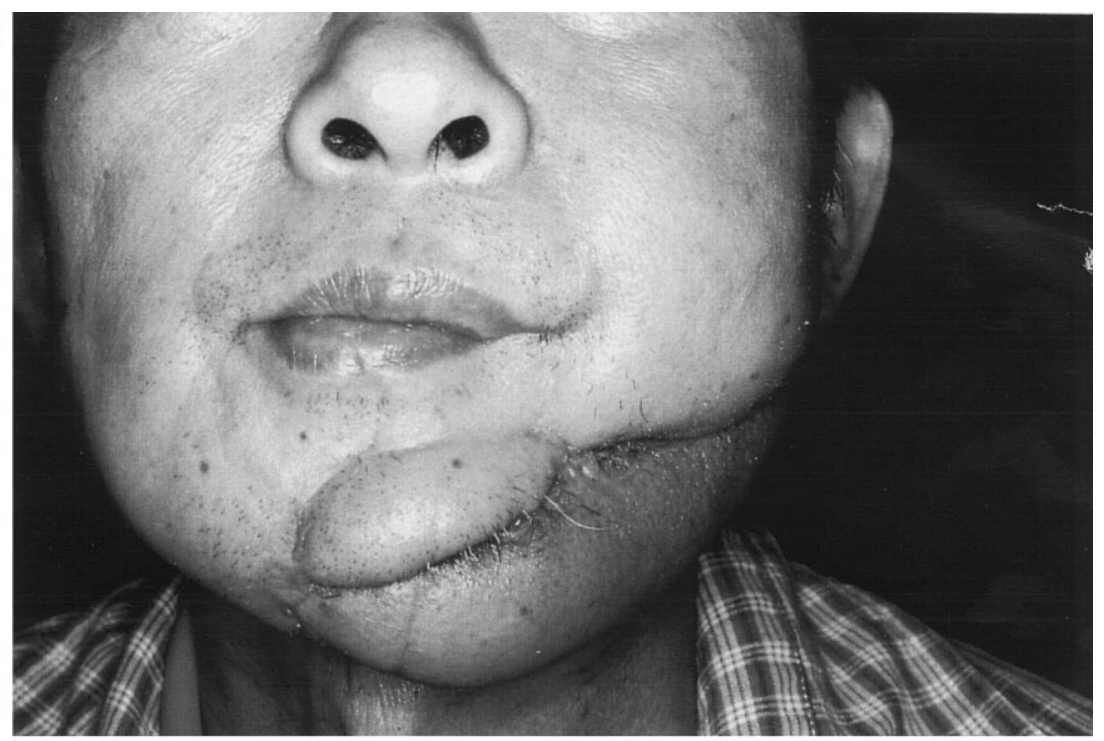

(f)

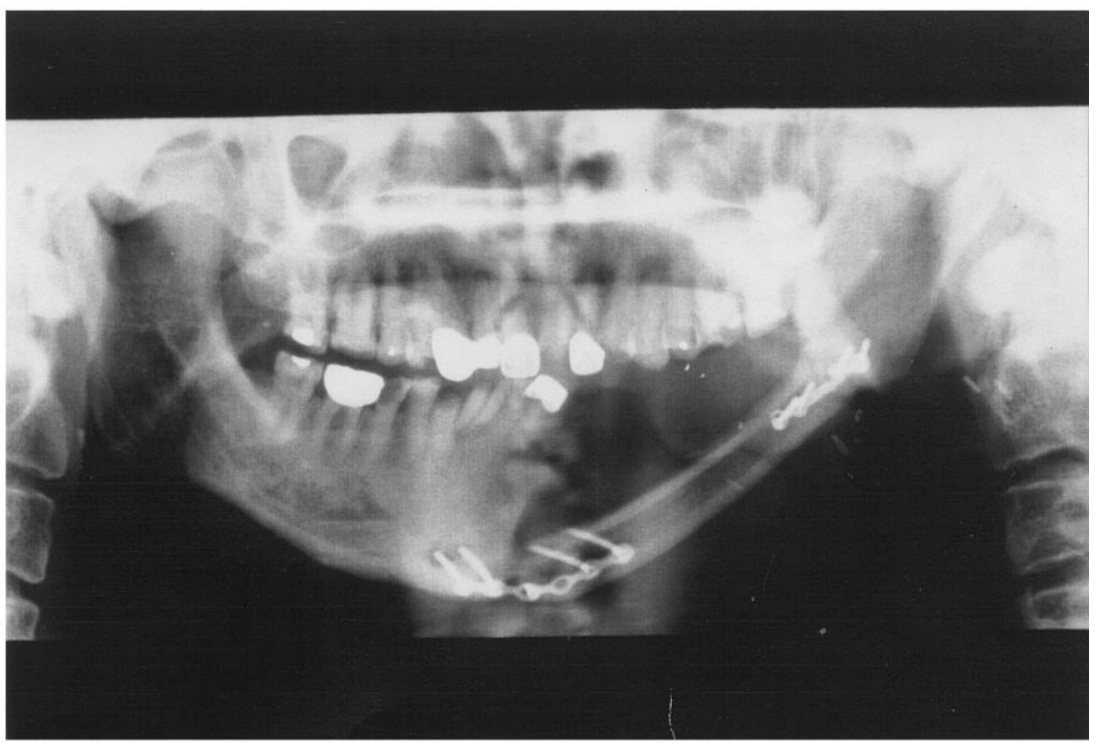

Fig. 1 (continued).

shaping the bone graft. The fibular osteotomy is done after division of the vascular pedicle. Careful subperiosteal dissection allows adequate exposure of the osteotomy sites while maintaining periosteal circulation to the fibula. Two to four osteotomies are made in the bone graft, and they are then fixed with miniplates.

\subsection{Graft insetting and revascularization}

Temporary intermaxillary fixation between the maxilla and remaining mandible helps precise inset of the bone graft. The shaped fibula is fixed to the ends of the remaining mandible with miniplates. After fixation, the osteocutaneous flap is revascularized using either the superior thyroid artery or the facial artery and a suitably sized vein nearby, with microsurgical techniques.

\section{Results}

3.1. A patient with poorly differentiated epidermoid carcinoma of the mouth floor

As shown in Fig. 1a, this 55-year-old man underwent resection of a $2.5-\mathrm{cm}$ poorly differentiated epidermoid carcinoma on floor of the mouth that included resection of the left mandible extending from angle to midline. This was performed in conjunction with a radical neck dissection.

Initial reconstruction consisted of a titanium reconstruction plate contoured to reestablish the mandibular arch. He received $6000 \mathrm{rad}$ of radiotherapy postoperatively. Al- 
though successfully oncologically treated, the patient had exposure of the plate through the skin, 18 months after the initial surgery (Fig. 1b). The plate had to be removed due to non-healing of the wound and persistent drainage.

Secondary reconstruction of the mandible consisted of a vascularized fibula osteocutaneous flap to reestablish the mandibular arch. The fibula osteocutaneous flap included a 12-cm fibula and an $18 \times 6-\mathrm{cm}$ skin island (Fig. 1c). The fibula was shaped to fit the remaining mandible with two osteotomies, and fixed with miniplates and screws (Fig. 1d). The skin island was used to close the external skin and soft tissue defect resulting from excision of the skin fistula and the surrounding scar tissue. After revascularization, the new mandible and skin island healed uneventfully (Fig. 1e). Fig. 1f showed postoperative X-ray radiography which demonstrated primary healing of the bone graft. The patient is currently free of disease, 3 years postoperatively.

\subsection{A patient with poorly differentiated osteogenic sar- coma of the left mandible}

As shown in Fig. 2a, this 20-year-old man underwent resection of the left mandible extending from the subcondylar region to the midline for a $6-\mathrm{cm}$ poorly differentiated osteogenic sarcoma of the mandible. This was performed in conjunction with a radical neck dissection.

Initial reconstruction consisted of a titanium tray contoured to reestablish the mandibular arch as arrowhead in Fig. 2b. He received 6500 rad of radiotherapy and a full course of chemotherapy postoperatively.

Two years after the initial surgery, the patient was free of disease. However, exposure of the hardware through the skin was noted as in Fig. 2c. The plate had to be removed after unsuccessful conservative treatment.

Secondary reconstruction of the mandible consisted of a vascularized fibula osteocutaneous flap to reestablish mandible continuity. Fig. $2 d$ showed the X-ray radiography of preoperation. The fibula osteocutaneous flap included a $15-\mathrm{cm}$ fibula and a $16 \times 6-\mathrm{cm}$ skin island (Fig. 2e).

The fibula was shaped to fit the remaining mandible with two osteotomies, and fixed with miniplates and screws as shown in Fig. 2f. The skin island was used to resurface the external skin and soft tissue defect resulting from adequate debridement. After revascularization, the new mandible and the skin island healed uneventfully (Fig. 2g). Fig. $2 \mathrm{~h}$ demonstrated a postoperative X-ray radiography. The patient is currently free of disease, 4 years postoperatively.

\subsection{The summary of the two studies}

All fibula osteocutaneous flaps survived. This was determined by the complete survival of the skin island and rapid healing of the transferred fibula on sequential X-ray studies. All osteotomy sites healed primarily.

Symmetry and contour of the reconstructed mandible were judged to be good in these two patients based on comparisons of preoperative and postoperative photographs and X-ray studies. Range of mouth opening was less than normal, but in neither patient was the compromise great enough to impair the ability to eat. These two patients are good candidates for osseointegrated dental implants, although none have been placed at the time of this writing.

The donor-site mobility was low. The donor-site over the leg was resurfaced with a skin graft. The leg was immobilized with plaster of Paris splint for 2 weeks. The initial leg edema resolved about 1 month after surgery, and the patient could ambulate freely after resolution of the leg

(a)

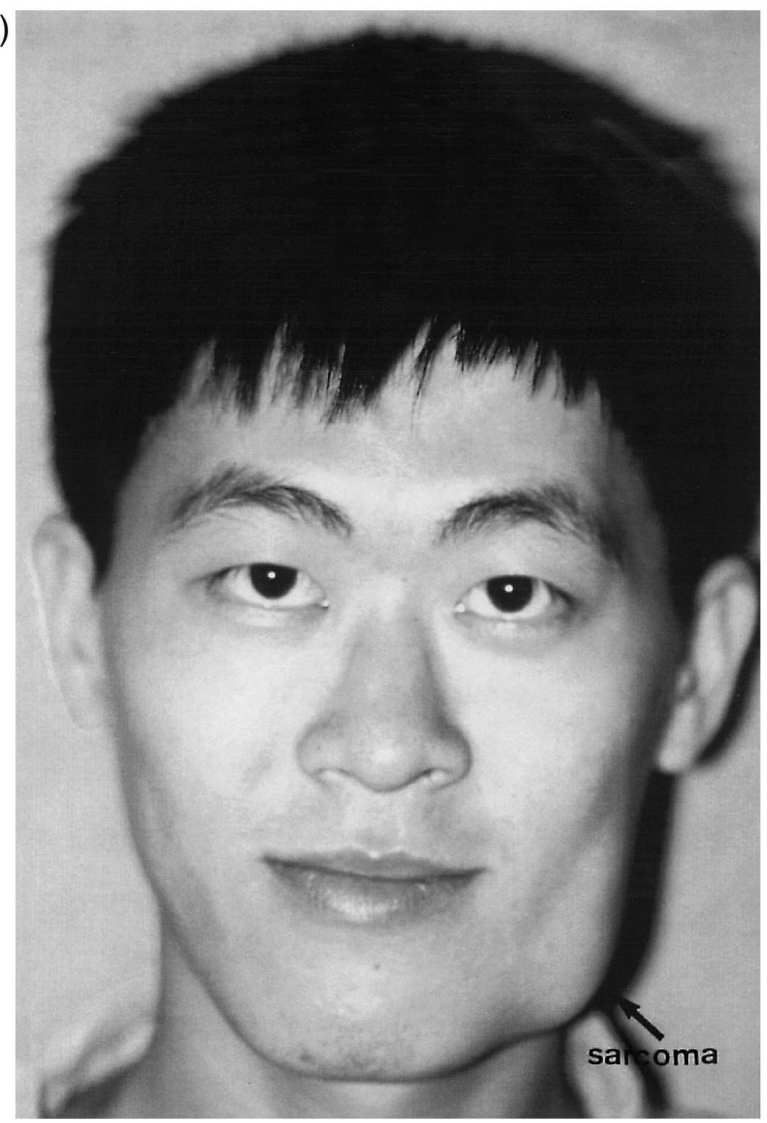

Fig. 2. (a) A patient with poorly differentiated osteogenic sarcoma of the left mandible (Arrow). (b) Initial reconstruction of the mandible with titanium tray. TT: Titanium tray. (c) Exposure of hardware (Arrow), 2 years after the initial surgery. (d) Preoperative X-ray. TT: Titanium tray. (e) Autogenous fibula osteocutaneous flap was harvested. AOF: Autogenous fibula osteocutaneous flap. (f) The fibula was osteomized to fit the remaining mandible and fixed with miniplates and screws (MPS). The skin island was used to resurface the external skin defect. (g) Postoperative uneventful healing of the skin island. (h) Postoperative X-ray showed primary healing of the bone graft. 


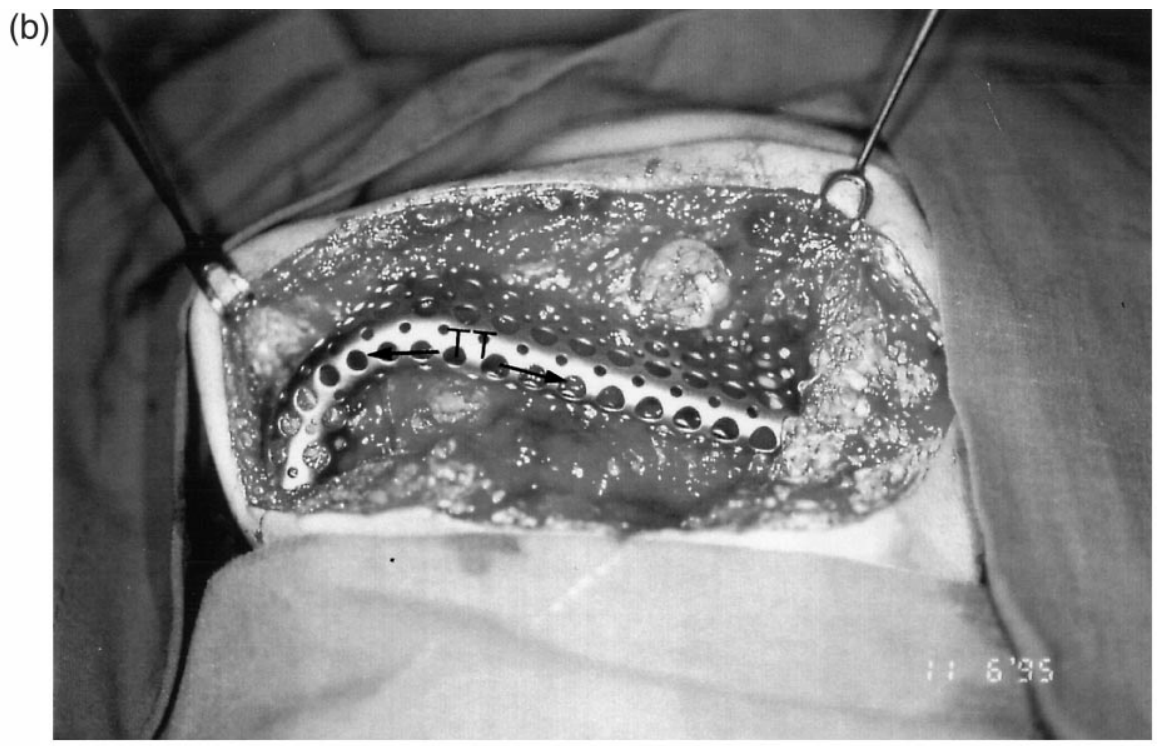

(c)

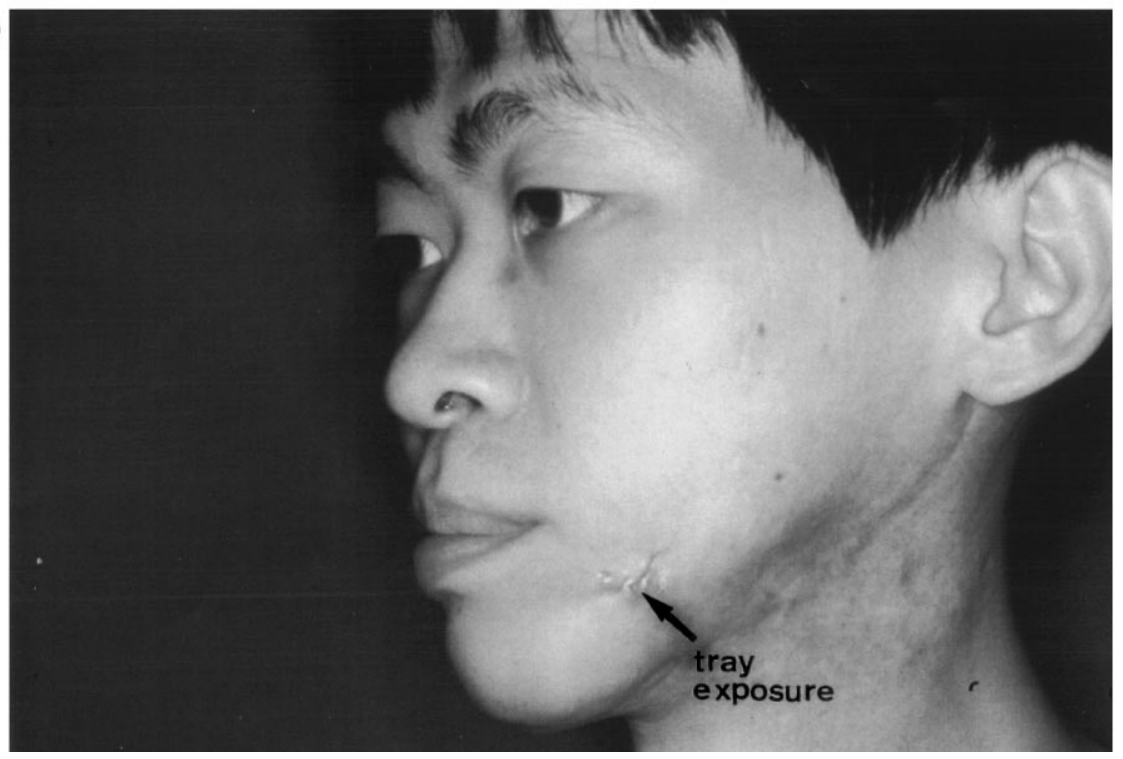

Fig. 2 (continued).

edema. There has not been any long-term donor-site morbidity observed in this study.

\section{Discussion}

The extent of the functional and aesthetic compromise, and the quality of life for patients undergoing surgical resection of oral cancer involving the mandible is dependent upon the amount of the tissue excised, as well as the method of immediate mandibular reconstruction. It became evident that more extensive resection, especially when the tongue, floor of mouth, and mandible were involved, resulted in less than satisfactory functional outcome in most cases.
Immediate reconstruction of the mandible after ablation surgery for oral cancer has been accomplished with both non-vascularized and vascularized bone graft. Experience with non-vascularized bone graft has been uniformly poor in patients who require postoperative irradiation. The hostile irradiation beds are incapable of providing metabolic demands for revascularization of the bone graft. The loss of bone graft can be expected under these circumstances. With the advent of microvascular bone transfer, immediate reconstruction of the mandible with live bone carrying its own blood supply becomes possible. The advantages of vascularized bone grafting, particularly in an irradiated bed, are well established. This technique, however, is not indicated in patients with advanced malignancy and a possible limited life expectancy. 
(d)

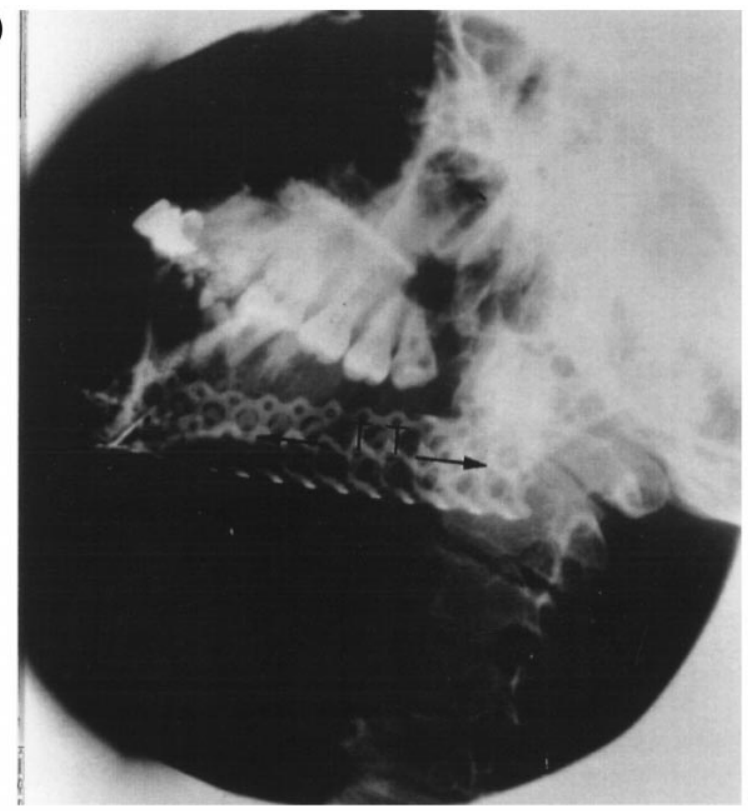

(e)

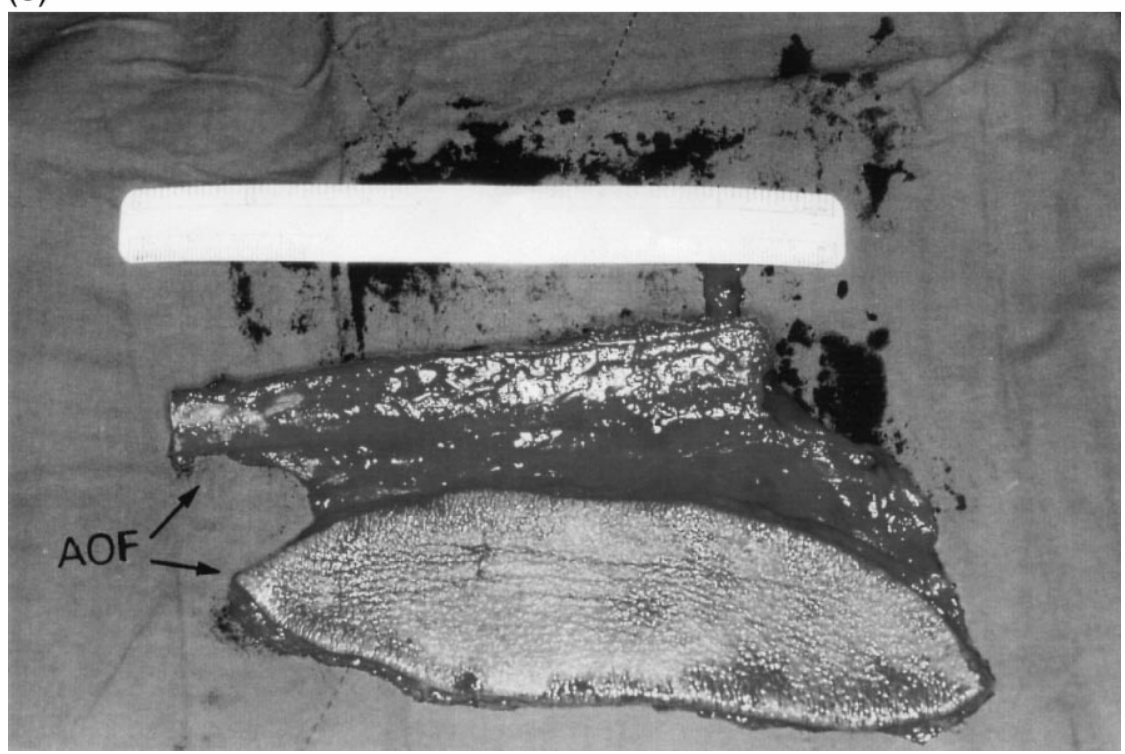

Fig. 2 (continued).

With the development of inert biocompatible materials capable of withstanding the masticatory forces and increased knowledge of the principle of internal fixation, alloplastic materials such as titanium plates and trays have emerged as alternatives for immediate mandibular reconstruction. The advantages of this approach include relative ease of contouring the implant, a decrease in donor-site disability and more rapid rehabilitation in those whose prospects for survival are poor and whose remaining time is precious.

Concern has been addressed with perturbation of irradiation when a titanium plate or tray was in place. It has long been long known that when metals are in the path of $\mathrm{X}$-ray or gamma ray beams, the dose pattern is altered and regions of increased or decreased doses are established [18-21]. It has been suggested that these metals may cause problems such as dose enhancement and tumor shielding. However, Gullane [22] has shown that the radiation dose at the metal-bone interface increased only $15 \%$ at the commonly used 6-MV energy level, and the excess tissue dose scatter extending into the surrounding tissue was only 1.1 $\mathrm{mm}$, which is clinically insignificant.

Another concern for the use of alloplastic material in mandibular reconstruction is its long-term stability. Longterm stability is directly related to the nature of the interface between the bone and the fixation screws of the plate. Stability of a metal can be more predictable if osseointegration can be achieved. For this reason, Raveh et al. [23] 
(f)

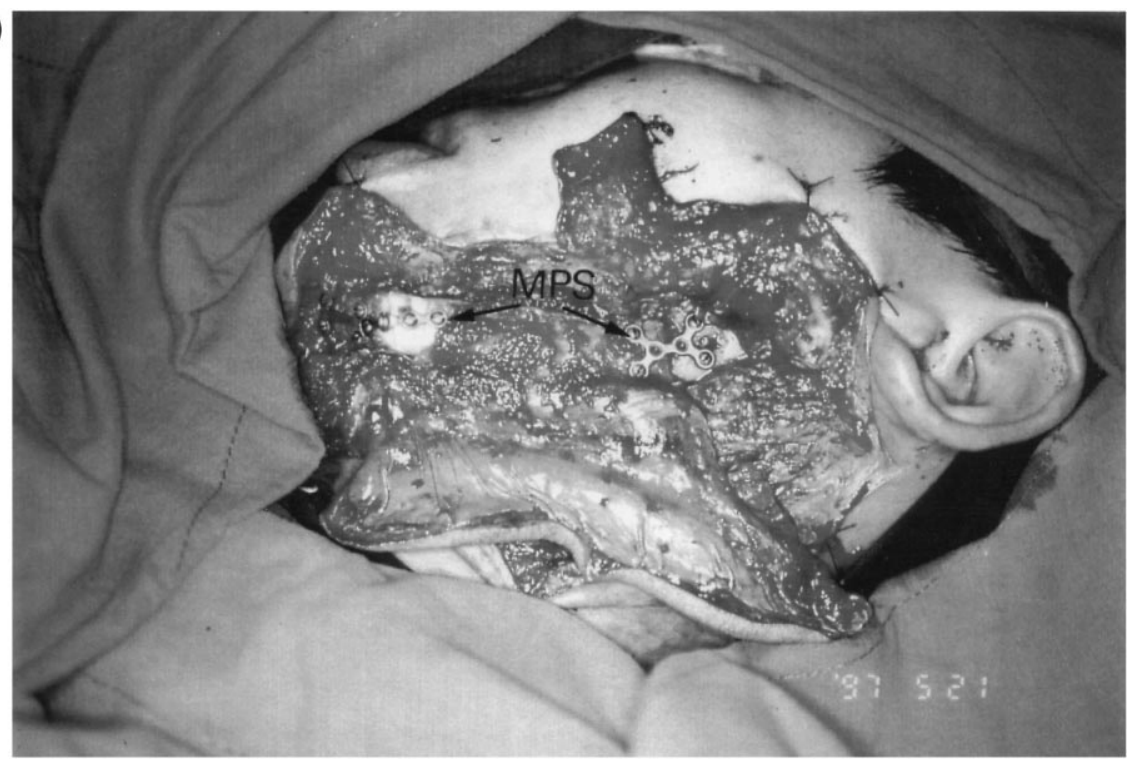

(g)

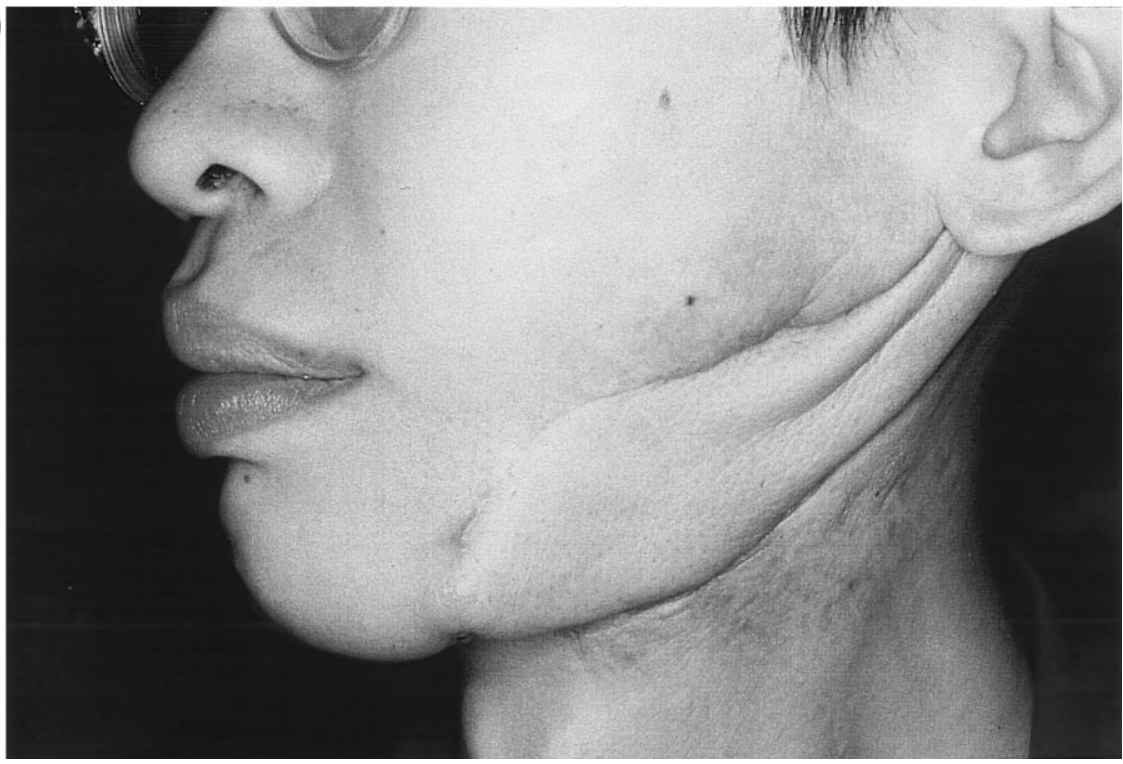

Fig. 2 (continued).

have used a titanium reconstruction plate with hollow screws that permitted proliferation of bone into the hollow screw in the reconstruction of mandibular defects following tumor resection. Subsequent clinical experiences reveal a very acceptable success rate [24-27]. More recently, Irish et al. [28] used the solid titanium screw rather than the hollow screw for applying the titanium plate. The advantage of using the solid screw is that it is easier to remove should plate failure occur, and with the use of the expansion bolt of screw, direct bone-screw union occurs rather than the fibrous bone-screw interface seen with a non-titanium screw.

Although the titanium plate or tray per se did not create problems, failures mainly resulted from the breakdown of the overlying soft tissue after irradiation with exposure and extrusion of the alloplastic material. The late detrimental effects of radiation to skin and soft tissue were well-established and include decreasing vascularity and inhibiting fibroblast activity [29,30]. Those effects often result in overlying skin necrosis with poor wound healing which can occur months or even years after radiotherapy. Most of the exposed alloplastic materials have to be removed and will result in invalid oral function. It is our philosophy that patients with advanced oral malignancy are best managed by immediate alloplastic mandibular reconstruction after cancer ablation surgery. In these patients, vascularized bone graft is reserved for rescuing the oral function should the alloplastic material fail or secondary bone reconstruction is necessary should the patient survive the test of time, which is usually $18-24$ months after the initial surgery. 
(h)

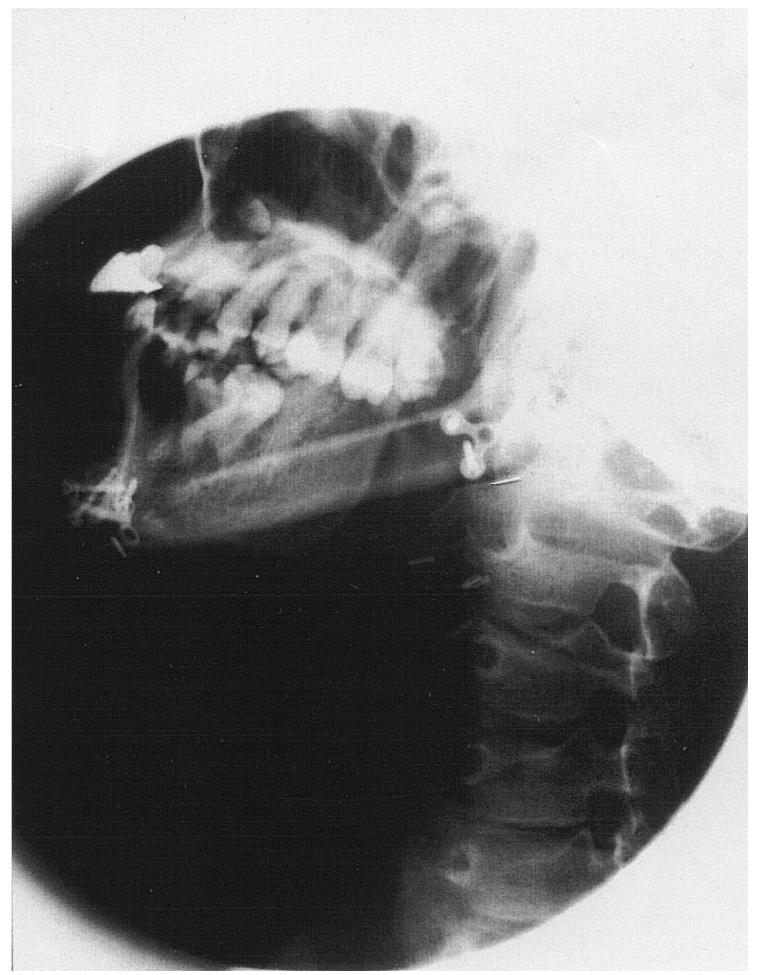

Fig. 2 (continued).

This is due to the fact that despite our best efforts, a significant number of these patients will succumb to the malignancy within 2 years of treatment.

A variety of vascularized bones has been used for mandibular reconstruction using microsurgical techniques. Current choices include ilium, radius, scapula, and fibula. The fibula is the most recently developed vascularized bone graft for mandible reconstruction [9,17,31-33]. The proven advantages are numerous: (1) No length limitation in adults, a straight $20-26-\mathrm{cm}$ fibular segment can be harvested; (2) Large diameter of vessels of sufficient length which facilitates microsurgical vessel reanastomosis; (3) Periosteal blood supply is abundant which permits multiple osteotomies and flexible contouring to fit the mandibular defect; (4) Adequate bone height and width to support osseointegrated dental implants; (5) Reliable skin island which can be used to resurface intraoral or extraoral defects; (6) Low donor-site morbidity. In our medical center, the fibula osteocutaneous flap is now the first choice if vascularized bone graft is indicated to reconstruct the mandible.

\section{Conclusion}

In summary, we feel that the three-dimensional, bendable, titanium plate or tray is a reasonable material for immediate mandible reconstruction after surgical resection of advanced oral malignancy. Vascularized bone graft is reserved for those patients with alloplastic material failure. We were able to rescue the oral function after alloplastic material failure with vascularized fibula osteocutaneous flap in irradiated patients. This approach can avoid subjecting the advanced oral cancer patient to an unnecessary extensive mandible reconstructive procedure.

\section{References}

[1] A.K. Adamo, R.L. Szal, Timing, results, and complications of mandibular reconstructive surgery: report of 32 cases, J. Oral Surg. 37 (1979) 755-763.

[2] B. Leipzing, C.W. Cummings, The current status of mandibular reconstruction using autogenous frozen mandibular grafts, Head Neck Surg. 6 (1984) 992-996.

[3] L.T. Ostrup, J.M. Fredrickson, Reconstruction of mandibular defects after radiation using free living bone graft transferred by microvascular anastomosis: an experimental study, Plast. Reconstr. Surg. 55 (1975) 563-572.

[4] M.J. Duncan, R.T. Manktelow, R.M. Zuker, I.B. Rosen, Mandibular reconstruction in the radiated patient: the role of osteocutaneous free-tissue transfer, Plast. Reconstr. Surg. 76 (1985) 829-840.

[5] G.I. Taylor, R.K. Daniel, Aesthetic aspects of microsurgery: composite tissue transfer to the face, Clin. Plast. Surg. 8 (1981) 333-339.

[6] D.S. Soutar, W.S. Widdowson, Immediate reconstruction of the mandible using a vascularized segment of radius, Head Neck Surg. 8 (1986) 232-246.

[7] D.D. Jewer, J.B. Boyd, R.T. Manktelow, R.M. Zuker, I.B. Rosen, P.J. Gullane et al., Orofacial and mandibular reconstruction with the iliac crest free falp: a review of 60 cases and a new method of classification, Plast. Reconstr. Surg. 84 (1989) 391-402.

[8] W.M. Swartz, J.C. Banis, E.D. Newton, S.S. Ramasastry, N.F. Jones, R. Acland, The osteocutaneous scapular flap for mandibular and maxillary reconstruction, Plast. Reconstr. Surg. 77 (1986) 531545.

[9] D.A. Hidalgo, Fibula free flap: a new method of mandible reconstruction, Plast. Reconstr. Surg. (1989) .

[10] J.M. Chow, Primary mandibular reconstruction using the A-O reconstruction plate, Laryngoscope 96 (1986) 768-773.

[11] P.J. Gullane, H. Holmes, Mandibular reconstruction: new concepts, Arch. Otolaryngol., Head Neck Surg. 112 (1986) 714-719.

[12] I.D. Papel, J.C. Price, H.K. Kashima, M.E. Jones, Compression plates in the treatment of advanced anterior floor or mouth carcinoma, Laryngoscope 96 (1986) 722-725.

[13] D.W. Klotch, J. Prein, Mandibular reconstruction using AO plates, Am. J. Surg. 154 (1987) 384-388.

[14] M.A. Schusterman, G.P. Reece, S.S. Kroll, M.E. Weldon, Use of the AO plate for immediate mandibular reconstruction in cancer patients, Plast. Reconstr. Surg. 88 (1991) 588-593.

[15] J. Davidson, B. Boyd, P.J. Gullane, L. Rotstein, J. Freeman, R. Manktelow et al., A comparison of the results following oromandibular reconstruction using a radial forearm flap with either radial bone or a reconstruction plate, Plast. Reconstr. Surg. 88 (1991) 201-208.

[16] J.B. Boyd, Use of reconstruction plates in conjunction with soft-tissue free flaps for oromandibular reconstruction, Clin. Plast. Surg. 21 (1994) 69-77.

[17] F.C. Wei, H.C. Chen, C.C. Chuang, M.S. Noordhoff, Fibular osteoseptocutaneous flap: anatomic study and clinic application, Plast. Reconstr. Surg. 78 (1986) 191-199.

[18] H.C. Schwartz, M. Wollin, D.L. Leake, A.R. Kagan, Interface radiation dosimetry in mandibular reconstruction, Arch. Otolaryngol. 105 (1979) 293-295. 
[19] M. Thatcher, A. Kuten, J. Helman, D. Laufer, Perturbation of cobalt-60 radiation doses by metal objects implants during oral and maxillofacial surgery, J. Oral Maxillofac. Surg. 42 (1984) 108-110.

[20] N. Scher, D. Poe, F. Kuchnir, C. Reft, R. Weichselbaum, W.R. Panje, Radiotherapy of the resected mandible following stainless steel plate fixation, Laryngoscope 98 (1988) 561-563.

[21] K.R. Postlethwaite, J.G. Philips, S. Booth, J. Shaw, A. Slater, The effects of small plate osteosynthesis on postoperative radiotherapy, Br. J. Oral Maxillofac. Surg. 27 (1989) 375-378

[22] P.J. Gullane, Primary mandibular reconstruction: analysis of 64 cases and evaluation of interface radiation dosimetry on bridging plates, Laryngoscope 101 (1991) 1-24.

[23] J. Raveh, H. Stich, F. Sutter, R. Greiner, New concepts in the reconstruction of mandibular defects following tumor resection, J. Oral Maxillofac. Surg. 41 (1983) 3-16.

[24] J. Raveh, H. Stitch, F. Sutter, R. Greiner, Use of the titanium-coated hollow screw and reconstruction plate system in bridging of lower jaw defects, J. Oral Maxillofac. Surg. 42 (1984) 281-294.

[25] S. Hellem, J. Olofsson, Titanium-coated hollow screw and reconstruction plate system (THORP) in mandibular reconstruction, J. Cranio-Max-Fac. Surg. 16 (1988) 173-183.

[26] T. Vuillemin, J. Raveh, F. Sutter, Mandibular reconstruction with the titanium hollow screw reconstruction plate (THORP) system: evaluation of 62 cases, Plast. Reconstr. Surg. 82 (1988) 804-814.

[27] D.W. Klotch, J. Gump, L. Kuhn, Reconstruction of mandibular defects in irradiated patients, Am. J. Surg. 160 (1990) 396-398.

[28] J.C. Irish, P.J. Gullane, R.W. Gilbert, D.H. Brown, B.D. Birt, J.B. Boyd, Primary mandibular reconstruction with the titanium hollow screw reconstruction plate: evaluation of 51 cases, Plast. Reconstr. Surg. 96 (1995) 93-99.

[29] S. Ariyan, T. Krizek, Radiation effects: biologic and surgical consideration, in: J.G. McCarthy (Ed.), Plastic Surgery, W.B. Saunder, Philadephia, 1990, pp. 831-848.

[30] N. Janjan, M. Michael, M.A. Schusterman, Therapeutic principles and options in radiation oncology, Plast. Reconstr. Surg. 96 (1995) $1463-1473$.

[31] D.A. Hidalgo, Fibula free flap mandibular reconstruction, Clin. Plast. Surg. 21 (1994) 25-35.

[32] F.C. Wei, C.S. Seah, Y.C. Tsai, S.J. Liu, M.S. Tsai, Fibular osteoseptocutaneous flap for reconstruction of composite mandibular defects, Plast. Reconstr. Surg. 93 (1994) 294-304.

[33] D.A. Hidalgo, A. Rekow, A review of 60 consecutive fibula free flap mandible reconstruction, Plast. Reconstr. Surg. 96 (1995) 585596. 